\title{
AVALIAÇÃO DA SELETIVIDADE DE INSETICIDAS A TRICHOGRAMMA SPP. (HYMENOPTERA: TRICHOGRAMMATIDAE) EM DIFERENTES HOSPEDEIROS
}

\section{R.M. Goulart ${ }^{1}$, S.A. De Bortoli ${ }^{1}$, R.T. Thuler ${ }^{1}$, D. Pratissoli ${ }^{2}$, C.L.T.P. Viana ${ }^{1}$, H.X.L. Volpe ${ }^{1}$}

${ }^{1}$ Universidade Estadual Paulista, Faculdade de Ciências Agráriase Veterinárias, Departamento deFitossanidade, Laboratório de Biologia e Criação de Insetos, Via de Acesso Prof. Paulo Donato Castellane, s/no, CEP14884-900, Jaboticabal, SP, Brasil. E-mail: rm_goulart@yahoo.com

\section{RESUMO}

\begin{abstract}
Objetivou-se estudar os efeitos de inseticidas químicos sobre Trichogramma pretiosum e Trichogramma exiguum em diferentes hospedeiros, utilizando a metodologia do Laboratório de Biologia e Criação de Insetos (LBCI), baseada na metodologia proposta pela IOBC/WPRS para estudos de seletividade. Os inseticidas utilizados foram: triflumurom, $(20 \mathrm{~mL} / 100 \mathrm{~L})$, etofenproxi, $(47 \mathrm{~mL} / 100 \mathrm{~L})$ e endossulfam $(750 \mathrm{~mL} / 100 \mathrm{~L})$, como testemunha foi utilizada água destilada. Utilizou-se delineamento inteiramente casualisado em esquema fatorial $4 \times 3$ (inseticidas $\times$ hospedeiros) em 20 repetições. Nos testes utilizaram-se cerca de 100 ovos de Anagasta kuehniella, delimitados em uma área de $0,2 \mathrm{~cm}^{2}, 30$ ovos de Spodoptera frugiperda e 30 ovos de Plutella xylostella por repetição, todos fixados em cartolina, que foram imersas em caldas químicas. Avaliou-se o número de ovos parasitados, a porcentagem de parasitismo, porcentagem de emergência, longevidade e razão sexual das gerações $\mathrm{F}_{1}$ e $\mathrm{F}_{2}$ de T. pretiosum e T. exiguum. Endossulfam foi extremamente tóxico aos parasitóides, inibindo o parasitismo em todos os hospedeiros. Etofenproxi demonstrou menor seletividade aos parasitóides. Triflumurom foi seletivo aos parasitóides quando se utilizou ovos de S. frugiperda e de P. xylostella.
\end{abstract}

PALAVRAS-CHAVE: Controle biológico, Trichogramma pretiosum, Trichogramma exiguum, produtos fitossanitários, efeitos tóxicos.

\section{ABSTRACT}

EVALUATION OF THE SELECTIVITY OF INSECTICIDES TO TRICHOGRAMMA SPP. (HYMENOPTERA: TRICHOGRAMMATIDAE) IN DIFFERENT HOSTS. The aim of this study was to evaluate the selectivity of pesticides to T.pretiosum and T. exiguum in different hosts. The tests were carried out using the LBCI(Laboratório de Biologia e Criação de Insetos) methodology based on the IOBC/WPRS methodology for selectivity studies. The hosts used were Anagasta kuehniella, Spodoptera frugiperda and Plutella xylostella eggs. The analysis of the selectivity was carried out for the products triflumuron $(20 \mathrm{~mL} / 100 \mathrm{~L})$, etofenproxi $(47 \mathrm{~mL} / 100 \mathrm{~L})$ and endosulfan $(750 \mathrm{~mL} / 100$ $\mathrm{L})$, with distilled water used as a control. Parasitized eggs, percentage of parasitism, percentage of emergence, longevity and sex ratio were evaluated in generations $\mathrm{F}_{1}$ and $\mathrm{F}_{2}$. Endosulfan was the most harmful, inhibiting the parasitism in all hosts. Etofenproxi showed low selectivity to parasitoids. Triflumuron was selective to parasitoid species when eggs of natural hosts were used.

KEY WORDS: Biological control, Trichogramma pretiosum, Trichogramma exiguum, pesticides, toxic effects.

\section{INTRODUÇÃO}

Para bons resultados em programas de Manejo Integrado de Pragas (MIP)éimportanteque os produtos fitossanitários eficientes contra as espécies-praga sejam seletivos aos insetos não-alvos (Degrande; Gomez, 1990; Faleiro et al., 1995, Reis et al., 1998).
Dentre os inimigos naturais, destacam-se os parasitóides do gênero Trichogramma que são cosmopolitasealimentam-se deinsetos-praga em diferentes cultivos (PINTO, 1997). Para o Brasil são registradas as ocorrências de 25 espécies distribuídas por quase todas as regiões (QuERINO; ZucCH, 2003). Trichogramma pretiosum Riley (Hym.: Trichogrammatidae) é a mais

\footnotetext{
${ }^{2}$ Universidade Federal do Espírito Santo, Centro de Ciências Agrárias, Departamento de Fitotecnia, Vitória, ES, Brasil.
} 
amplamente distribuída, tendo sido relatada em 18 diferentes hospedeiros e 13 culturas (ZuCCHI;MONTEIRO, 1997). Segundo esses autores, Trichogramma exiguum Pinto \& Platner (Hym.: Trichogrammatidae) é a segunda espécie com o maior número de hospedeiros no continente sul-americano. A ocorrência desta espécie foi recentemente relatada no Brasil em plantios de tomateiro no Distrito de Piaçu, em Muniz Freire, ES (Oliveira et al., 2003).

Com o intuito de selecionar produtos fitossanitários que pudessem ser utilizados em associação com o controle biológico e permitissem a otimização do controle de lepidópteros praga (CAMPBELL et al., 1991; Wetzel; DickLer, 1994), foi criado, em 1974, pela "International Organization for Biological and Integrated Control of Noxious Animals and Plants (IOBC), o "Working Group of Pesticides and Benefical Organisms".

No Brasil foram realizados vários estudos relacionados à seletividade de inseticidas a T. pretiosum (CARvalho et al., 2001a; CARvAlHo et al., 2002), porém, ainda são escassos estudos que utilizam a técnicapadrão proposta pela IOBC para estudos de seletividade de produtos fitossanitários a inimigos naturais (RoCHA; CARVALHO, 2004). É necessário averiguar se a metodologia proposta pela IOBC/WPRS pode ser considerada padrão, tendo em vista que para o gênero Trichogramma é usada apenas uma espécie desse parasitóide, T. cacoeciae Marchal (Hym.: Trichogrammatidae) e um hospedeiro alternativo, Sitotroga cerealella (Oliv.,1819) (Lep.: Gelechiidae), independentemente das condições bióticas e abióticas serem distintas nas diferentes regiões onde se utilizam parasitóides do gênero, para o controle de diversas pragas em inúmeras culturas.

O objetivo deste estudo foi avaliar os efeitos de inseticidas sobre Trichogramma spp. em diferentes hospedeiros usando metodologia adaptada ou baseada da IOBC.

\section{MATERIAL E MÉTODOS}

O ensaio foi realizado no Laboratório de Biologia e Criação de Insetos (LBCI) do Departamento de Fitossanidade da Faculdade de Ciências Agrárias e Veterinárias de Jaboticabal - UNESP, sob temperatura de $25 \pm 1^{\circ} \mathrm{C}$, umidade relativa de $70 \pm 10 \%$ e fotofase de 12 horas.

As espécies do parasitóide de ovos T. pretiosum, linhagem Tp-8, e T. exiguum, linhagem Te-1, utilizadas na experimentação, fazem parte da coleção de Trichogramma do Laboratório de Entomologia do Centro de Ciências Agrárias da Universidade Federal do Espírito Santo (LE-CCA/UFES).
Como hospedeiros foram utilizados ovos da traçadas-farinhas, Anagasta kuehniella (Zeller, 1879)(Lep.: Pyralidae) (hospedeiro alternativo), adquiridos semanalmentejunto à empresa BUG Agentes de Controle Biológico (Piracicaba, SP); ovos da lagarta-do-cartucho do milho, Spodoptera frugiperda(J.E.Smith) (Lep.: Noctuidae) (hospedeiro natural), obtidos no Laboratório de Ecologia Aplicada do Departamento de Fitossanidade da FCAV/UNESP e ovos da traça-dascrucíferas, Plutella xylostella(L., 1758) (Lep.:Plutellidae) (hospedeiro natural), procedentes da criação estoque do LBCI.

Foram utilizados, como tratamentos, os inseticidas e suas respectivas dosagens: triflumurom (Certero ${ }^{\circledR}$ - benzoiluréia), $20 \mathrm{~mL} / 100 \mathrm{~L}$ de água, etofenproxi (Trebon 100SC ${ }^{\circledR}$ - éter-piretróide), 47 mL/100 L de água e endossulfam (Thiodan $\mathrm{CE}^{\circledR}$ - clorociclodieno), $750 \mathrm{~mL} / 100 \mathrm{~L}$ deágua, medidas essas recomendadas pelos fabricantes como dosagens máximas a serem aplicadas no campo, além da testemunha, composta por água destilada.

Utilizou-se delineamento inteiramente casualisado em esquema fatorial $4 \times 3$ (inseticidas $x$ hospedeiros) em 20 repetições por tratamento. Nos testes realizados com o hospedeiro A. kuehniella foram utilizados, aproximadamente, 100 ovos por cartela, delimitados em uma área de $0,2 \mathrm{~cm}^{2}$, para $S$. frugiperda e P. xylostella utilizou-se 30 ovos por cartela, todos os ovos foram colados com goma arábica $(15 \%)$ diluída em água em cartelas de cartolina azul celeste $(0,4 \times 2,0$ $\mathrm{cm})$. Em testes preliminares observou-se que o número de ovos parasitados por fêmea em ovos deP.xylostella e $S$. frugiperda não ultrapassou a quantidade oferecida no atual experimento.

As cartelas foram imersas nas diferentes caldas por 5 segundose, após secagem por 2 horas em capela de exaustão, foram expostas à oviposição por 24 horas em tubos de Duran que abrigavam uma fêmea (recém emergida) de parasitóide, em seu interior, sendo esse processo realizado somente para geração $F_{1}$. Para os referidos tratamentos, nas primeiras 24 hapós a emergência dos adultos, cartelas idênticas às citadas anteriormente, porém, sem contato com os inseticidas, foram oferecidas aos descendentes, por $24 \mathrm{~h}$, para avaliação dos efeitos dos inseticidas sobre a geração $F_{2}$.

Foi empregada a metodologia de THuler (2006), utilizando-se preceitos da IOBC, quais sejam: contato, exposição e aeração do ambiente. Para tanto, foram utilizadas mini-câmaras de exaustão, construídas especialmente para a realização dos testes. Cada mini-câmara foi composta por placas do tipo ELISA ${ }^{\circledR}$ que foram perfuradas e os furos cobertos com tecido tipo "voil" para se evitar a fuga dos insetos. As placas foram acopladas duas a duas, a uma distância de $2 \mathrm{~cm}$ uma da outra, através de faixas de cartolina presas 
com e fita adesiva. Externamente foram acopladas ponteiras de micropipetagem $(1.000 \mu \mathrm{L})$, com o ápice cortado, permitindo o encaixe da ponteira no poço da placa de ELISA ${ }^{\circledR}$. Posteriormente, tubos de Duran, contendo as cartelas e a fêmea do parasitóide,foram acoplados nas ponteiras. As mini-câmaras e as ponteiras foram cobertas com uma tira de borracha, tipo E.V.A (etil vinil acetato), preto, mantendo escuro o centro da câmara e clara a extremidade para que os insetos mantivessem contato com os ovos contaminados, haja vista que eles são atraídos pela luz.

Para promover a aeração foram utilizados mini compressores de ar (compressor de aquário), acoplados às câmaras, permitindo o fluxo contínuo de ar durante $24 \mathrm{~h}$.

Avaliou-se o número de ovos parasitados, porcentagem de parasitismo (considerando-se $100 \%$, o maior número de ovos parasitados observado nos tratamentos das testemunhas) porcentagem de emergência, longevidade e razão sexual dos parasitóides nas gerações $\mathrm{F}_{1} \mathrm{eF}_{2}$ de $T$. pretiosum e T. exiguum. Os dados obtidos foram submetidos à análise de variância e as médias dos tratamentos foram comparadas teste de Tukey $(5 \%)$, através do programa ESTAT/FCAVUNESP versão 2.0.

\section{RESULTADOS E DISCUSSÃO}

Endossulfam foi altamente tóxico a T. pretiosume T. exiguum, matando rapidamente os adultos deambas as espécies quando estes entravam em contato com ovos tratados, dos diferentes hospedeiros; tal fato pode ter ocorrido devido à ação dos gases desse organoclorado. A morte prematura dos insetos impediu que a avaliação dos parâmetros biológicos fosse realizada. Ao contrário do observado nesse estudo, JACAS; VinUELA (1993), utilizando-se da metodologia padrão proposta pela IOBC/WRPS (HASSAN et al., 2000), constataram a seletividade de endossulfam para diferentes espécies de Trichogramma e cinco espécies de predadores.
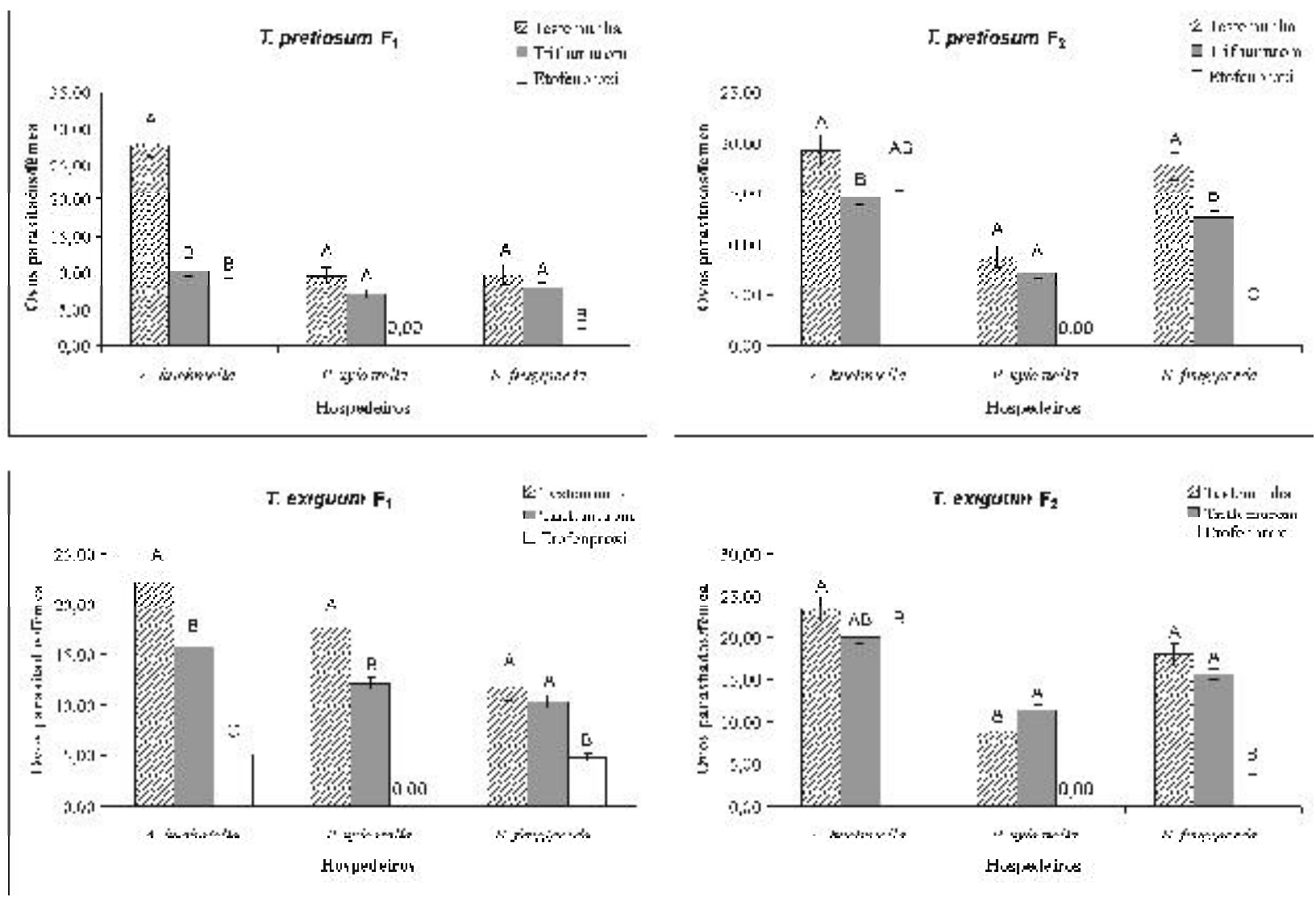

Fig. 1 - Número de ovos parasitados por fêmea $( \pm \mathrm{EP})$ da geração $\mathrm{F}_{1}$ e $\mathrm{F}_{2}$ de Trichogramma pretiosum e de Trichogramma exiguum em ovos de três hospedeiros, tratados com diferentes inseticidas. Médias seguidas pela mesma maiúscula na coluna não diferem entre si pelo teste de Tukey $(p=0,05)$. 
Em relação ao número de ovos parasitados, as médias obtidas nas testemunhas do hospedeiro $A$. kuehniella foram as maiores em relaçãoas testemunhas dos hospedeiros naturais, nas gerações $\mathrm{F}_{1}$ e $\mathrm{F}_{2}$ de $T$. exiguum e T.pretiosum(Fig.1), oqueindica um provável condicionamento pré imaginal do parasitóide, adquirido durante o desenvolvimentolarval, o que, segundo COBERT (1985), pode ocorrer quando um parasitóide é criado por várias gerações no hospedeiro alternativo. Embora o maior número dos ovos parasitados não tenha sido dos hospedeiros naturais, T. exiguum e $T$. pretiosum, em suas gerações descendentes $\left(\mathrm{F}_{2}\right)$, revelaram uma tendência à adaptação em ovos de $S$. frugiperda (testemunha), após a criação desses parasitóides nesse hospedeiro por uma geração (Fig. 1).

Segundo WAJNBERG et al. (1989), SCHMidt (1991) e PAK et al. (1986), a aceitação pelo hospedeiro não depende somente de herança genética, mas também de características herdadas ao longo das gerações. Essa afirmação pode explicar o maior número de ovos parasitados nas testemunhas com A. kuehniella, por ambas as espécies de parasitóides, nas duas gerações, pois a criações foram mantidas em A. kuehniella.
Referente a ação dos inseticidas aplicados em ovos dos hospedeiros contendo os parasitóides, etofenproxi mostrou-se prejudicial às duas espécies de parasitóides, que parasitaram menor número de ovos do hospedeiro alternativo em $\mathrm{F}_{1}$, por seguinte diferindo-se em quase todos os hospedeiros em $\mathrm{F}_{2}$. Em ovos de P. xylostella, triflumurom mostrou-se seletivo T. pretiosum e prejudicial T. exiguum em $\mathrm{F}_{1}$, etofenproxi inibiu o parasitismo das duas espécies impedindo a avaliação de $\mathrm{F}_{2}$ neste hospedeiro. $\mathrm{O}$ número de ovos de $S$. frugiperda foi afetado por triflumurom e etofenproxi diferindo da testemunha. Em $\mathrm{F}_{1} \mathrm{e}_{2}$ de ambas as espécies houve diferenças de parasitismo entre triflumurom e o etofenproxi (Fig. 1).

$\mathrm{Na}$ geração $\mathrm{F}$ de $\mathrm{T}$. pretiosum os inseticidas triflumurom e etofenproxi reduziram em $39 \%$ e 44,4\%, respectivamente, a porcentagem de parasitismo em relação a testemunha, quando ovos de A. kuehniella foram tratados por imersão com esses produtos; já para a geração $\mathrm{F}_{1}$ de $T$. exiguum, a redução na porcentagem de parasitismo foi de $14,8 \%$ e $50,2 \%$, respectivamente (Fig. 1).

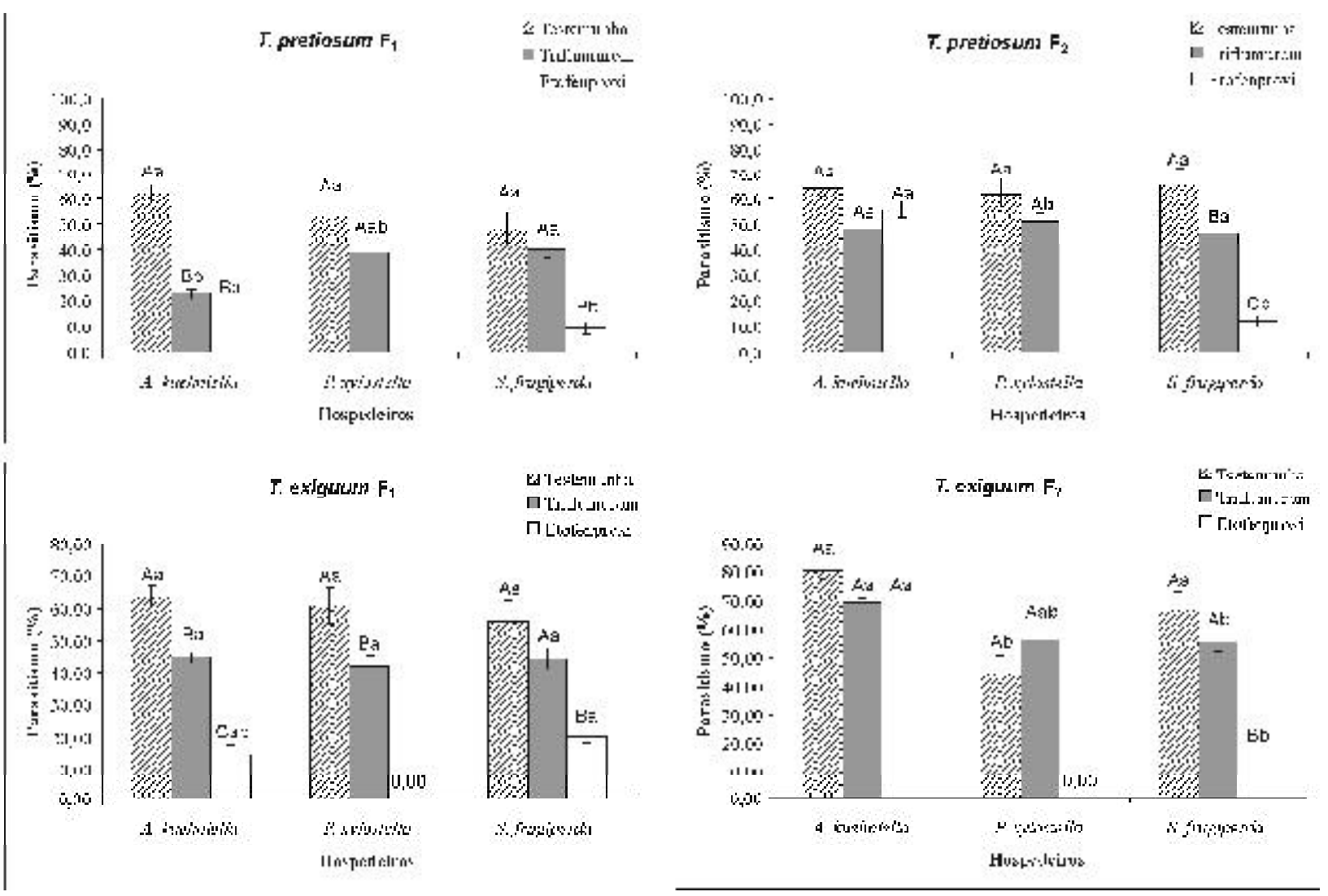

Fig. 2 - Porcentagem de parasitismo $( \pm \mathrm{EP})$ da geração $\mathrm{F}_{1}$ e $\mathrm{F}_{2}$ de Trichogramma pretiosum e de Trichogramma exiguumem ovos de três hospedeiros, tratados com diferentes inseticidas. Médias seguidas pela mesma letra minúscula na linha e maiúscula na coluna não diferem entre si pelo teste de Tukey $(p=0,05)$. 
A ação do triflumurom para T. pretiosum foi verificada por CARVALHO etal. (2001b), que observaram redução no parasitismo em $26 \%$ para a linhagem $\mathrm{L}_{10}$, de Venda Nova do Imigrante - ES, em ovos de A. kuehniella. Essas diferenças de respostas biológicas entre populações de T. pretiosum estão relacionadas principalmente com as características de seus locais de origem (Bleicher, 1985; BRUNner et al., 2001). Além disso, CARVAlHo et al. (1998) sugeriu que a maior exposição de populações desse parasitóidea determinado inseticida pode ter selecionadoindivíduos mais tolerantes, diferenciando as respostas das populações em condições de laboratório.

Triflumurom não afetou a porcentagem de parasitismo de T.pretiosum $\left(\mathrm{F}_{1}\right)$ em ovos deP.xylostella e $S$. frugiperda, não ocorrendo diferenças significativas em relação à testemunha desses hospedeiros. Em ovos de A. kuehniella e P. xylostella, a porcentagem de parasitismo da geração $F_{1}$ de $T$. exiguum foi afetada negativamente, com diferenças estatísticas em relação à testemunha. A geração $\mathrm{F}_{2}$ de $T$. exiguum não foi afetada pela ação desse produto, independente do hospedeiro utilizado, enquanto que $\mathrm{F}_{2}$ de T.pretiosum foi afetada somente em ovos de $S$. frugiperda, onde se observou redução da porcentagem de parasitismo em relação à testemunha com o uso de triflumurom (Fig.2) .

Etofenproxi prejudicou a ação dos parasitóides da geração $\mathrm{F}_{1}$ de $T$. pretiosum e T. exiguum em ovos de $A$. kuehniella e inibiu a oviposição de, $P$. xylostella, o que impediu que a porcentagem de parasitismo e os demais parâmetros fossem avaliados tanto para $\mathrm{F}_{1}$ quanto para $\mathrm{F}_{2}$ nas duas espécies estudadas. Volpe et al. (2006) relataram que este inseticida causou repelência aT.exiguum. Em ovos de S. frugiperda foram constatadas reduções de
$38,7 \%$ e $33,3 \%$ na porcentagem de parasitismo por $T$. pretiosum $\left(\mathrm{F}_{1}\right)$ e T. exiguum $\left(\mathrm{F}_{1}\right)$, respectivamente, em relação à testemunha. Tais fatos podem também ser atribuídos à ação repelente do etofenproxi, dado que JACOBS etal.(1984) e CARVALHOetal.(2001b) relataramque fêmeas de Trichogramma evitaram o contato com ovos tratados com inseticidas piretróides, tanto em laboratório quanto em casa de vegetação.

TAKADA et al. (2001) relataram alta mortalidade, em todos os estágios de desenvolvimento, deT.dendrolimi Matsumura (Hym: Trichogrammatidae), quando expostos a ovos do hospedeiro Mamestra brassicae (L.,1758) (Lepidoptera: Noctuidae) tratados com etofenproxi. Observou-se que a porcentagem de parasitismo na geração $\mathrm{F}_{2}$ de T. exiguum diferiu apenas em ovos do hospedeiro $P$. xylostella, onde foi obtido o menor valor em relação aos outros hospedeiros, enquanto que na geração $\mathrm{F}_{2}$ de T. pretiosum não foram encontradas diferenças significativas entre os hospedeiros (Fig. 2).

Com o uso de triflumurom, a geração $\mathrm{F}_{1}$ de $T$. pretiosum mostrou o melhor resultado em ovos do hospedeiro natural S. frugiperda, com $40 \%$ de parasitismo, diferindo significativamente somente de A. kuehniella (Fig. 2), e a $\mathrm{F}_{2}$ apresentou melhores resultados em ovos do hospedeiro P. xylostella com $50,9 \%$ de parasitismo, ocorrendo diferença significativa em relação à A. kuehniella e S. frugiperda (Fig. 2).

$\mathrm{NaF}_{1}$ deT. exiguum não houve diferença significativa entre os hospedeiros para o parâmetro avaliado, havendo seletividade de triflumurom aos parasitóides ena $\mathrm{F}_{2} \mathrm{o}$ hospedeiro A. kuehniella diferiu significativamente de $S$. frugiperda, apresentando o maior valor para porcentagem de parasitismo (69,3\%) (Fig. 2).

Tabela 1 - Porcentagem de emergência e longevidade $( \pm \mathrm{EP})$ da geração $\mathrm{F}_{1}$ de Trichogramma pretiosum e de Trichogramma exiguum em ovos de três hospedeiros, tratados com diferentes inseticidas.

\begin{tabular}{|c|c|c|c|c|c|c|}
\hline \multirow[t]{3}{*}{ Inseticidas } & \multicolumn{6}{|c|}{ Hospedeiros } \\
\hline & \multicolumn{3}{|c|}{ Emergência (\%) } & \multicolumn{3}{|c|}{ Longevidade (dias) } \\
\hline & A. kuehniella & P. xylostella & S. frugiperda & A. kuehniella & P. xylostella & S. frugiperda \\
\hline \multicolumn{7}{|l|}{ T. pretiosum } \\
\hline Testemunha & $100,0 \pm 0,00 \mathrm{Aa}$ & $100,0 \pm 0,00 \mathrm{Aa}$ & $100,0 \pm 0,00 \mathrm{Aa}$ & $4,3 \pm 0,61 \mathrm{Ab}$ & $7,6 \pm 1,07 \mathrm{Aa}$ & $4,9 \pm 0,76 \mathrm{Ab}$ \\
\hline Triflumurom & $100,0 \pm 0,00 \mathrm{Aa}$ & $100,0 \pm 0,00 \mathrm{Aa}$ & $100,0 \pm 0,00 \mathrm{Aa}$ & $5,4 \pm 0,82 \mathrm{Ab}$ & $8,1 \pm 0,76 \mathrm{Aa}$ & $4,1 \pm 0,54 \mathrm{Ab}$ \\
\hline Etofenproxi & $100,0 \pm 0,00 \mathrm{Aa}$ & $100,0 \pm 0,00 \mathrm{Aa}$ & $100,0 \pm 0,00 \mathrm{Aa}$ & $4,4 \pm 0,67 \mathrm{Aa}$ & - & $4,4 \pm 0,93 \mathrm{Aa}$ \\
\hline Endossulfam & - & - & - & - & - & - \\
\hline \multicolumn{7}{|l|}{ T. exiguum } \\
\hline Testemunha & $97,4 \pm 1,00 \mathrm{Aa}$ & $100,0 \pm 0,00 \mathrm{Aa}$ & $100,0 \pm 0,00 \mathrm{Aa}$ & $4,4 \pm 0,50 \mathrm{ABa}$ & $4,6 \pm 0,67 \mathrm{Aa}$ & $4,2 \pm 0,43 \mathrm{Aa}$ \\
\hline Triflumurom & $99,3 \pm 0,51 \mathrm{Aa}$ & $100,0 \pm 0,00 \mathrm{Aa}$ & $98,9 \pm 0,78 \mathrm{Aa}$ & $5,4 \pm 0,50 \mathrm{Aa}$ & $3,4 \pm 0,47 \mathrm{Ab}$ & $4,1 \pm 0,36 \mathrm{Aab}$ \\
\hline Etofenproxi & $97,0 \pm 1,98 \mathrm{Aa}$ & - & $97,8 \pm 1,10 \mathrm{Aa}$ & $3,8 \pm 0,28 \mathrm{Ba}$ & - & $4,2 \pm 0,32 \mathrm{Aa}$ \\
\hline Endossulfam & - & - & - & - & - & - \\
\hline
\end{tabular}

Médias seguidas pela mesma letra minúscula na linha e maiúscula na coluna não diferem entre si pelo teste de Tukey $(p=0,05)$. Dados para longevidade transformados por $(x+0,5)^{1 / 2}$. 
Tabela 2 - Porcentagem de emergência e longevidade $( \pm \mathrm{EP})$ da geração $\mathrm{F}_{2}$ de Trichogramma pretiosum e de Trichogramma exiguum em ovos de três hospedeiros, tratados com diferentes inseticidas.

\begin{tabular}{|c|c|c|c|c|c|c|}
\hline \multirow[t]{3}{*}{ Inseticidas } & \multicolumn{6}{|c|}{ Hospedeiros } \\
\hline & \multicolumn{3}{|c|}{ Emergência (\%) } & \multicolumn{3}{|c|}{ Longevidade (dias) } \\
\hline & A. kuehniella & P. xylostella & S. frugiperda & A. kuehniella & P. xylostella & S. frugiperda \\
\hline \multicolumn{7}{|l|}{ T. pretiosum } \\
\hline Testemunha & $100,0 \pm 0,00 \mathrm{Aa}$ & $100,0 \pm 0,00 \mathrm{Aa}$ & $100,0 \pm 0,00 \mathrm{Aa}$ & $5,1 \pm 0,59 \mathrm{Aa}$ & $6,1 \pm 0,84 \mathrm{Aa}$ & $5,4 \pm 0,75 \mathrm{Aa}$ \\
\hline Triflumurom & $100,0 \pm 0,00 \mathrm{Aa}$ & $100,0 \pm 0,00 \mathrm{Aa}$ & $100,0 \pm 0,00 \mathrm{Aa}$ & $4,7 \pm 0,77 \mathrm{Aa}$ & $3,0 \pm 0,29 \mathrm{Ba}$ & $3,2 \pm 0,63 \mathrm{Aa}$ \\
\hline Etofenproxi & $100,0 \pm 0,00 \mathrm{Aa}$ & $100,0 \pm 0,00 \mathrm{Aa}$ & $100,0 \pm 0,00 \mathrm{Aa}$ & $6,0 \pm 0,67 \mathrm{Aa}$ & - & $3,4 \pm 0,97 \mathrm{Ab}$ \\
\hline Endossulfam & - & - & - & - & - & - \\
\hline \multicolumn{7}{|l|}{ T. exiguum } \\
\hline Testemunha & $98,6 \pm 0,58 \mathrm{Aa}$ & $100,0 \pm 0,00 \mathrm{Aa}$ & $100,0 \pm 0,00 \mathrm{Aa}$ & $2,1 \pm 0,74 \mathrm{Aab}$ & $2,0 \pm 0,54 \mathrm{Ab}$ & $2,6 \pm 0,93 \mathrm{ABa}$ \\
\hline Triflumurom & $99,2 \pm 0,60 \mathrm{Aa}$ & $99,6 \pm 0,47 \mathrm{Aa}$ & $99,1 \pm 0,58 \mathrm{Aa}$ & $2,2 \pm 0,51 \mathrm{Aab}$ & $1,8 \pm 0,45 \mathrm{Ab}$ & $2,4 \pm 0,38 \mathrm{Ba}$ \\
\hline Etofenproxi & $100,0 \pm 0,00 \mathrm{Aa}$ & - & $97,8 \pm 1,76 \mathrm{Aa}$ & $2,1 \pm 0,43 \mathrm{Ab}$ & - & $3,0 \pm 1,33 \mathrm{Aa}$ \\
\hline Endossulfam & - & - & - & - & - & - \\
\hline
\end{tabular}

Médias seguidas pela mesma letra minúscula na linha e maiúscula na coluna não diferem entre si pelo teste de Tukey $(p=0,05)$. Dados para longevidade transformados por $(x+0,5)^{1 / 2}$.

Tabela 3 - Razão sexual $( \pm \mathrm{EP})$ das gerações $\mathrm{F}_{1} \mathrm{eF}_{2}$ de Trichogramma exiguum em ovos de três hospedeiros, tratados com diferentes inseticidas.

\begin{tabular}{lccr}
\hline Inseticidas & \multicolumn{2}{c}{ Hospedeiros } \\
\cline { 2 - 4 } & & Razão sexual & S. frugiperda \\
\cline { 2 - 4 } & A. kuehniella & P. xylostella & $0,90 \pm 0,02 \mathrm{Aa}$ \\
\hline $\mathrm{F}_{1}$ & & $0,82 \pm 0,03 \mathrm{Aa}$ \\
Testemunha & $0,86 \pm 0,01 \mathrm{Aab}$ & $0,81 \pm 0,02 \mathrm{Ab}$ & $0,82 \pm 0,04 \mathrm{Aa}$ \\
Triflumurom & $0,84 \pm 0,02 \mathrm{Aa}$ & $0,84 \pm 0,4 \mathrm{Aa}$ & - \\
Etofenproxi & $0,91 \pm 0,03 \mathrm{Aa}$ & - & $0,83 \pm 0,03 \mathrm{Ba}$ \\
Endossulfam & - & - & $0,86 \pm 0,01 \mathrm{ABa}$ \\
$\mathrm{F}_{2}$ & & $0,88 \pm 0,03 \mathrm{Aa}$ & $0,92 \pm 0,04 \mathrm{Aa}$ \\
Testemunha & $0,88 \pm 0,02 \mathrm{Aa}$ & $0,87 \pm 0,02 \mathrm{Aa}$ & - \\
Triflumurom & $0,91 \pm 0,01 \mathrm{Aa}$ & - & - \\
Etofenproxi & $0,88 \pm 0,01 \mathrm{Aa}$ & - &
\end{tabular}

Médias seguidas pela mesma letra minúscula na linha e maiúscula na coluna não diferem entre si pelo teste de Tukey $(\mathrm{p}=0,05)$.

O uso de etofenproxi inibiu ou reduziu drasticamente a porcentagem de parasitismo de ambas as espécies de parasitóides nos hospedeiros testados; apenas na $\mathrm{F}_{2}$ para $A$. kuehniella a porcentagem de parasitismo não foi afetada negativamente (Fig. 2).

Triflumurom eetofenproxi não afetaram a porcentagem de emergência dos indivíduos nas $\mathrm{F}_{1}$ e $\mathrm{F}_{2}$ de $T$. pretiosum e T. exiguum, obtendo-se $100 \%$ de emergência para T. pretiosum em todos os tratamentos, independente do hospedeiro utilizado (Tabelas 1 e 2). Resultados semelhantes foram encontrados por Pratissoli et al. (2004) em ovos de A. kuehniella, e por
Beserra; Parra (2004) em ovos de S. frugiperda, sendo de 93,0\% e 93,8\%, respectivamente.PereIra etal.(2004) encontraram porcentagem deemergência inferior para a mesma espécie de parasitóide, em ovos deP.xylostella $(84,8 \%)$. T. exiguum apresentou porcentagens de emergência que não diferiram estatisticamente entre si (Tabelas 1 e 2).

A longevidade de T. pretiosum $\left(\mathrm{F}_{1}\right)$ não foi afetada pela ação dos inseticidas, não havendo diferenças significativas em relação à testemunha, em todos os hospedeiros (Tabela 1). As $F_{1}$ e $F_{2}$ de $T$. exiguum diferiram da testemunha quando etofenproxi foi 
utilizado em ovos de A. kuehniella (Tabela 1). Esse efeito não foi observado em ovos de $S$. frugiperda tratados com esse produto, obtendo-se o maior valor absoluto (3,0 dias) na geração $F_{2}$ de $T$. pretiosum (Tabela 2).

Ao se comparar a longevidade dos parasitóides nos hospedeiros estudados constatou-se que a maior longevidade obtida foi com T. pretiosum $\left(\mathrm{F}_{1}\right)$ no tratamento com triflumurom em ovos de P. xylostella $(8,1$ dias), valor superior ao da testemunha (Tabela 1 ). Para T. exiguum $\left(\mathrm{F}_{1}\right)$, no tratamento com triflumurom, o maior valor obtido (5,4 dias) foi observado em ovos de A. kuehniella, valor também superior ao da testemunha (Tabela 1).

Na longevidade da geração $\mathrm{F}_{2}$ de $T$. exiguum, os indivíduos provenientes de ovos de A. kuehniella e $P$. xylostella mostraram pequena diferença em relação à S. frugiperda (Tabela2) notratamentocom triflumurom. Em T. pretiosum $\left(\mathrm{F}_{2}\right)$, a longevidade dos indivíduos foi afetada com etofenproxi em ovos de $S$. frugiperda (Tabela 2).

LEWIS et al. (1976) também encontraram maior longevidade em ovos de A. kuehniella do que naqueles de S. cerealella para Trichogramma sp. BAI et al. (1995) mostraram que para T. brassicae Bezdenko, 1968, T. minutum Riley, 1879 eT. nr. sibiricumSorokina, 1984 (Hym: Trichogrammatidae) ocorre variação na longevidade entre diferentes espécies de $(8,6$ - 9,2 dias, de 10,2 - 11,7 dias e de 8,3 - 12,4 dias, respectivamente).

A razão sexual de $T$. pretiosum foi igual a 1, com ausência de machos nas duas gerações e em todos os hospedeiros, independentemente dos inseticidas utilizados (Tabela 3). Tal fenômeno pode ser atribuídoà presença de microrganismos como Wolbakia, que inibem o desenvolvimento de machos (STOUTHAMERet al., 1993; Pereira et al., 2004) e ao fato de, a criação do parasitóide ter sido mantida por várias gerações em laboratório.

$\mathrm{Na} \mathrm{F}_{2}$ de T. exiguum, observou-se que no procedimento onde ovos de $S$. frugiperda foram tratados com etofenproxi ocorreu aumento no número de machos. (Tabela 3). O' BRIEN et al. (1985) também constataram alterações na razão sexual de parasitóides quando expostos a inseticidas, sendo que estes autores verificaram que descendentes adultos de Bracon mellitor Say, 1836 (Hym: Braconidae), tratados com LC Le $_{5}$ azinfós-metileclordimeforme, mostraram uma maior relação fêmea: macho, quando comparados a descendentes não tratados (Moura et al., 2004).

Com relação aos hospedeiros, observou-se diferença significativa apenas nas testemunhas da geração $F_{1}$ de T. exiguum. (Tabela 3). Clausen (1939) e PARRA; ZuCCHI (1997) afirmaram que a qualidade do hospedeiroéo principal fator que influência na razão sexual.

\section{CONCLUSÕES}

- O inseticida endossulfam foi extremamente tóxico aos parasitóides T. exiguum e T. pretiosum, inibindo totalmente a oviposição em todos os hospedeiros avaliados.

- Para etofenproxi e triflumurom foram obtidas respostas diferentes de seletividade em função dos hospedeiros, indicando que o uso de apenas um hospedeiro como padrão para os testes subestima ou superestima os efeitos dos inseticidas sobre os parasitóides.

- Triflumurom foi seletivo.

- Etofenproxi foi moderadamente tóxico às espécies T. pretiosum e T. exiguum.

\section{AGRADECIMENTOS}

Ao APECOLAB/FCAV-UNESP, Jaboticabal, SP, na pessoa do Prof. Dr. Odair Aparecido Fernandes, pelo fornecimento dos ovos de S. frugiperda, utilizados no experimento.

\section{REFERÊNCIAS}

BAI, B.; COBANOGLU, S.; SMITH, S.M. Assessment of Trichogramma species for biological control of forest lepidopteran defoliators. Entomologia Experimentalis et Applicata, v.75, n.2, p.135-145, 1995.

BESERRA, E.B.; PARRA, J.R.P. Biologia e parasitismo de Trichogramma atopovirilia Oatman \& Plantner e Trichogramma pretiosum Riley (Hymenoptera: Trichogrammatidae) em ovos de Spodoptera frugiperda (J. E. Smith) (Lepidoptera: Noctuidae). Revista Brasileira de Entomologia, v.48, n.1, p.119-126, 2004.

BLEICHER, E. Biologia e exigências térmicas de populações de Trichogramma (Hym: Trichogrammatidae). 1985. 80f. Tese (Doutorado em Agronomia - Entomologia Agrícola) - Escola Superior de Agricultura "Luiz de Queiroz", Universidade de São Paulo, Piracicaba. 1985.

BRUNNER, J.F.; DUNLEY, J.E.; DOERR, M.D.; BEERS, E.H. Effect of pesticides on Colpoclypeus florus (Hymenoptera: Eulophidae) and Trichogramma platneri (Hymenoptera: Trichogrammatidae), parasitoids of leafrollers in Washington. Journal of Economic Entomology, v.94, n.5, p.1075-1084, 2001.

CAMPBELL, C.D.; WALGENBACH, J.F.; KENNEDY, G.C. Effect of parasitoids on lepidopterous pests in insecticide-treated and untreated tomatoes in western North Carolina. Journal of Economic Entomology, v.84, n.6, p.1662-1667, 1991. 
CARVALHO, C.F.; SOUZA, B.; SANTOS, T.M. Predation capacity and reproduction potential of Chrysoperla externa (Hagen) (Neuroptera. Chrysopidae) fed on Alabama argillacea (Hûbner) eggs. Acta Zoologica Fennica, v.209, n.1, p.83-86, 1998.

CARVALHO, G.A.; PARRA, J.R.P.; BAPTISTA, J.C. Seletividade de alguns produtos fitossanitários a duas linhagens de Trichogramma pretiosum Riley, 1879 (Hymenoptera: Trichogrammatidae). Ciência e Agrotecnologia, v.25, p.583-591, 2001a.

CARVALHO, G.A.; PARRA, J.R.P; BAPTISTA, G.C. de. Impacto de produtos fitossanitários utilizados na cultura do tomateiro na fase adulta de duas linhagens de Trichogramma pretiosum Riley (1879) (Hymenoptera: Trichogrammatidae). Ciência e Agrotecnologia, v.25, n.3, p.560-568, 2001b.

CARVALHO, G.A.; REIS, P.R.; MORAES, J.C.; FUINI, L.C.; ROCHA, L.C.D.; GOUSSAIN, M.M. Efeitos de alguns inseticidas utilizados na cultura do tomateiro (Lycopersicon esculentum Mill.) a Trichogramma pretiosum Riley, 1879 (Hymenoptera: Trichogrammatidae). Ciência e Agrotecnologia, v.26, n.6, p.1160-1166, 2002.

CLAUSEN, C.P. The effect of host size upon the sex ratio of hymenopterous parasites and its relation to methods of rearing and colonization. Journal of the New York Entomological Society, v.47, p.1-9, 1939.

COBERT, S. A. Insect chemosensory responses: a chemical legacy hypothesis. Ecological Entomology, v.10, p.143-153, 1985.

DEGRANDE, P.E.; GOMEZ, D.R.S. Seletividade de produtos químicos no controle de pragas. Agropecuária, v.7, p.8-13, 1990.

FALEIRO, F.G., PICANÇO, M.C.; PAULA, S.U.; BATALHA, V.C. Seletividade de inseticidas a Spodoptera frugiperda (J.E. Smith) (Lepidoptera: Noctuidae) e ao predador Doru luteipes (Scudder) (Dermaptera: Forficulidae). Anais da Sociedade Entomológica do Brasil, v.24, n.2, p.247-252, 1995.

HASSAN, S.A.; HALSALL, N.; GRAY, A.P.; KUEHNER, C.; MOLL, M.; BAKKER, F. M.; ROEMBKE, J.; YOUSEF, A.; NASR, F.; ABDELGADER, H.A. A laboratory method to evaluate the side effects of plant protection products on Trichogramma cacoeciae Marchal (Hym., Trichogrammatidae). In: CANDOLFI, M.P.; BLÜMEL, S.; FORSTER, R.; BAKKER, F.M.; GRIMM, C.; HASSAN, S.A.; HEIMBACH, U.; MEAD-BRIGGS, M.A.; REBER, B.; SCHMUCK, R.; VOGT, H. (Eds.). Guidelines to evaluate side-effects of plant protection products to non-target arthropods. Reinheim: IOBC/WPRS, 2000. p.107-119.

JACAS, J.A.; VIÒUELA, E. Los efectos de los plaguicidas sobre los organismos benéficos en la agricultura. Phytoma, v.48, p.45-52, 1993.
JACOBS, R.J.; KOUSKOLESKAS, C.A; GROSS JUNIOR, H.R. Responses of Trichogramma pretiosum (Hymenoptera: Trichogrammatidae) to residues of permethrin and endosulfan. Environmental Entomology, v.13, n.2, p.355-358, 1984.

LEWIS, W.J.; GROSS JR., H.R.; PERKINS, W.D.; KNIPLING, E.F.; VOEGELÉ, J. Production and performance of Trichogramma reared on eggs of Heliothis zea and other hosts. Environmental Entomology, v.5, n.3, p.449-452, 1976.

MOURA, A.P.; CARVALHO, G.A.; RIGITANO, R.L.O. Efeito residual de novos inseticidas utilizados na cultura do tomateiro sobre Trichogramma pretiosum Riley, 1879 (Hymenoptera: Trichogrammatidae). Acta Scientiarum - Agronomy, v.26, n.2, p.231-237, 2004.

O'BRIEN, P.J.; ELZE, G.W.; VINSON, S.B. Toxicity of azinphosmethyl and chlordimeform to parasitoid Bracon mellitor (Hymenoptera: Braconidae): lethal and reproductive effects. Environmental Entomology, v.14, p.891-894, 1985.

OLIVEIRA, H.N.; PRATISSOLI, D.; COLOMBI, C.A.; ESPINDULA, M.C. Características biológicas de Trichogramma exiguum Pinto \& Platner em ovos de Corcyra cephalonica Stainton. Magistra, v.15, n.1, p.103105, 2003.

PAK, A.G.; BUIS, H.C.E.M.; HECK, I.C.C.; HERMANS, M.L.G. Behavioral variations among strains of Trichogramma spp.: host-age selection. Entomologia Experimentalis et Applicata, v.40, n.3, p.247-258, 1986.

PARRA, J.R.P.; ZUCCHI, R.A. Trichogramma e o controle biológico aplicado. Piracicaba: FEALQ, 1997. 324p.

PEREIRA, F.F.; BARROS, R.; PRATISSOLI, D.; PARRA, J.R.P. Biologia e exigências térmicas de Trichogramma pretiosum Riley e T. exiguum Pinto \& Platner (Hymenoptera: Trichogrammatidae) criados em ovos de Plutella xylostella (L.) (Lepidoptera: Plutellidae) Neotroprical Entomology, v.33, n.2, p.231-236, 2004.

PINTO, J.D. Taxonomia de Trichogrammatidae (Hymenoptera) com ênfase nos gêneros que parasitam Lepidoptera, In: PARRA, J.R.P.; ZUCCHI, R.A. (Ed.). Trichogramma e o controle biológico aplicado. Piracicaba: FEALQ, 1997. p.13-40.

PRATISSOLI, D.; HOLTZ, A.M.; GONÇALVES, J.R.; OLIVEIRA, R.C.; VIANNA, U.R. Características biológicas de linhagens de Trichogramma pretiosum, criadas em ovos de Sitotroga cerealella e Anagasta kuehniella. Horticultura Brasileira, v.22, n.3, p.562-565, 2004.

QUERINO, R.B.; ZUCCHI, R.A. O gênero Trichogramma (Hymenoptera: Trichogrammatidae) no Brasil. In: SIMPÓSIO DE CONTROLE BIOLÓGICO, 8., 2003, São Pedro. Resumos. São Paulo: SICONBIOL, 2003. p.131. 
REIS, P.R.; CHIAVEGATO, L.G.; MORAES, G.J.; ALVES E.B.; SOUSA, E.O. Seletividade de agroquímicos ao ácaro predadorIphiseiodes zuluagaiDenmark\&Muma (Acari: Phytoseiidae). Anais da Sociedade Entomológica do Brasil, v.27, n.2, p.65-274,1998.

ROCHA, L.C.D; CARVALHO, G.A. Adaptação da metodologia padrão da IOBC para estudos de seletividade com Trichogramma pretiosum Riley, 1879 (Hymenoptera: Trichogrammatidae) em condições de laboratório. Acta Scientiarum - Agronomy, v.26, n.3, p.315-320, 2004.

SCHMIDT, J.M. The role of physical factors in tritrophic interactions. Redia Giornale di Entomologia, v.124, p.43-87, 1991.

STOUTHAMER, R.; BREEUWER, J.A.J.; LUCK. R.F.; WERREN, J.H. Molecular identification of microorganisms associated with parthenogenesis. Nature, London, v.361, p.66-68, 1993.

TAKADA, Y.; KAWAMURA, S.; TANAKA, T. Effects of various insecticides on the development of the egg parasitoid Trichogramma dendrolimi (Hymenoptera: Trichogrammatidae). Journal of Economic Entomology, v.94, n.6, p.1340-1343, 2001.

THULER, R.T. Plutella xylostella (L.) (Lepidoptera: Plutellidae): táticas para o manejo integrado em brássicas. 2006. 79p. Tese (Doutorado em Entomologia Agrícola) - Faculdade de Ciências Agrárias e Veterinárias, UNESP, Jaboticabal, 2006.
VOLPE, H.X.L.; THULER, R.T.; DE BORTOLI, S.A.; VIANA, C.L.T.P.; GOULART, R.M. Repelência de Inseticidas para Trichogramma exiguum Pinto \& Platner (Hymenoptera: Trichogrammatidae). Biológico, São Paulo, v.68, n.2, 2006. , 2006. Suplemento 2. Trabalho apresentado na REUNIÃO ANUAL DO INSTITUTO BIOLÓGICO, 19., 2006, São Paulo. Resumo 207/002. Suplemento. Disponível em <http:/ / www.biologico. sp.gov.br/biologico/v68_supl_raib/207.PDF> Acesso em: 7 jan. 2007.

WAJNBERG, E.; PIZZOL, J.; BABAULT, M. Genetic variation in progeny allocation in Trichogramma maidis. Entomologia Experimentalis et Applicata, v.53, n.3, p.177-187, 1989.

WETZEL, C.; DICKLER, E. Side effects of sulphur and a natural pyrethroid on Trichogramma dendrolimi Matsumura (Hym., Trichogrammatidae) in apple orchards. In: VOGT, H. (Eds.). Side-effects of pesticides on beneficial organisms: comparison of laboratory, semifield and field results. IOBCMPRS Bulletin, v.17, n.10, p.123-132, 1994.

ZUCCHI, R.A, MONTEIRO, R.C. O gênero Trichogramma na América do Sul. In: PARRA, J.R.P.; ZUCCHI, R.A. (Ed.). Trichogramma e o controle biológico aplicado. Piracicaba: FEALQ, 1997. p.41-46.

Recebido em 4/3/07

Aceito em 24/3/08 